\title{
PRIMER REGISTRO DE PANOCHTHUS GRESLEBINI CASTELLANOS (XENARTHRA, GLYPTODONTIDAE, HOPLOPHORINI) PARA LA REGIÓN PAMPEANA, ARGENTINA
}

\author{
MARTÍN ZAMORANO \\ División Paleontología de Vertebrados, Museo de La Plata, Facultad de Ciencias Naturales y Museo, Universidad Nacional \\ de La Plata, CONICET, Paseo del Bosque s/n, 1900, La Plata, Argentina.marzamorano@fcnym.unlp.edu.ar \\ CRISTIAN OLIVA \\ Centro de Registro del Patrimonio Arqueológico y Paleontológico, Dirección Provincial de Patrimonio Cultural, Instituto \\ Cultural de la Provincia de Buenos Aires, Calle 50 N539, La Plata, Argentina.cristianoliva 78@yahoo.com.ar
}

\section{GUSTAVO J. SCILLATO-YANÉ,}

División Paleontología de Vertebrados, Museo de La Plata, Facultad de Ciencias Naturales y Museo, Universidad Nacional de La Plata, CONICET, Paseo del Bosque s/n, 1900, La Plata, Argentina. scillato@fcnym.unlp.edu.ar

\section{JOSÉ DARIVAL FERREIRA}

Programa de Pós-Graduação em Geociências, UFRGS, Campus do Vale, Porto Alegre, RS, Brasil. darival.fds@gmail.com

\author{
ALFREDO E. ZURITA \\ Centro de Ecología Aplicada del Litoral (CECOAL-CONICET) y Universidad Nacional del Nordeste. Ruta 5, \\ km 2,5, 3400, Corrientes, Argentina.aezurita74@yahoo.com.ar
}

\begin{abstract}
FIRST RECORD OF PANOCHTHUS GRESLEBINICASTELLANOS (XENARTHRA, GLYPTODONTIDAE, HOPLOPHORINI) FROM THE PAMPEAN REGION, ARGENTINA. Panochthus greslebini Castellanos (= P. rochai Paula Couto) has been considered an endemic taxon from northeastern Brazil, although in recent times some uncertain records have been postulated from southern Brazil and central-north Argentina (Santiago del Estero Province). In this contribution we report and describe a well preserved caudal tube (MMH-QUQ 09-03-01), from the late Pleistocene of the lower course of Quequén Salado river, Buenos Aires Province (Lujanian Age/Stage). This material is assigned to P. greslebini on the basis of the following characters: (i) presence of an apexian figure on the distal end of the caudal tube; (ii) on the dorsal view there is one apical figure distal to first dorsal-ventral pair, as in Panochthus subintermedius Castellanos (however, in P. subintermedius, the distal end is thin and less depressed than the material here reported). From a geographic perspective, this represents the southernmost record of the species, expanding its latitudinal distribution in more than $3000 \mathrm{~km}$. It was also noted that this material, in overall morphology and size, is the more similar to the holotype of P. greslebini than all previously known caudal tubes so far attributed to this species.
\end{abstract}

Key words: Argentina, Panochthus greslebini, caudal tube, Pampean Region.

RESUMO - Panochthus greslebini Castellanos (= P. rochai Paula Couto) tem sido tradicionalmente considerado um táxon endêmico do NE de Brasil. No entanto, nos últimos anos houve registros, ainda que com determinações duvidosas, para o Sul do Brasil e para a Província de Santiago del Estero, Argentina. Na presente contribuição se dá a conhecer e se descreve detalhadamente um tubo caudal completo, em bom estado de preservação (MMH-QUQ 09-03-01) atribuído a P. greslebini, procedente do Lujanense (Pleistoceno tardio) do curso inferior do rio Quequén Salado, Província de Buenos Aires. Este constitui o primeiro achado confiável da espécie para a Argentina e por sua vez o registro mais austral, ampliando notavelmente sua distribuição latitudinal. O material aqui apresentado exibe todas as características diagnósticas descritas para o holótipo, a saber: (i) possui uma figura apexiana no extremo distal; (ii) em vista dorsal se encontra, distalmente, uma única figura apical, localizada distalmente entre o primeiro par dorso-ventral, como em $P$. subintermedius Castellanos, mas neste último o extremo distal é o mais agudo e o tubo é menos deprimido. Do ponto de vista geográfico, representa o registro mais ao sul da espécie, ampliando sua distribuição latitudinal em mais de $3.000 \mathrm{~km}$. Verificou-se também que este material, em morfologia e tamanho global, é o mais semelhante ao holótipo de . greslebini do que todos os tubos caudais previamente conhecidos até agora atribuídos a esta espécie.

Palavras-chave: Argentina, Panochthus greslebini, região Pampeana, tubo caudal. 


\section{INTRODUCCIÓN}

Panochthus Burmeister, 1866 representa uno de los gliptodóntidos más abundantes y diversificados del Pleistoceno sudamericano, como así también uno de los cingulados de mayor tamaño (e.g. Fariña, 2001; Soibelzon et al., 2012). Recientes revisiones (Zamorano, 2012a,b; Zamorano et al., 2014; Porpino et al., 2014) reconocen seis especies válidas: (1) P. intermedius Lydekker, 1895, Ensenadense (Pleistoceno temprano tardío) de la ciudad de Buenos Aires, Argentina y Cochabamba, Bolivia; (2) P. subintermedius Castellanos, 1937, Ensenadense (Pleistoceno temprano tardío) de la ciudad de Buenos Aires, Argentina; (3) P. tuberculatus (Owen, 1845), Bonaerense-Lujanense (Pleistoceno medio-Pleistoceno tardío) de gran parte de Argentina, Uruguay, sur y centro de Bolivia y sur de Brasil; (4) P. frenzelianus Ameghino, 1889, Bonaerense (Pleistoceno medio-Pleistoceno tardío) de la Provincia de Buenos Aires, Argentina y los alrededores de Montevideo, Uruguay; (5) P. greslebini Castellanos, 1942 y (6) P. jaguaribensis Moreira, 1965, Pleistoceno del noreste de Brasil (Ameghino, 1889; Lydekker, 1895; Castellanos, 1937, 1942; Moreira, 1965; Hoffstetter, 1978, 1963, 1964; Mones \& Francis, 1973; Ubilla et al., 2004; Tauber \& Palacios, 2007; Kerber \& Oliveira, 2008; Tonni et al., 2009; Zurita et al., 2009; Zurita et al., 2011).

Panochthus greslebini y P. jaguaribensis son especies tradicionalmente consideradas endémicas del noreste de Brasil (Moreira, 1971; Bergqvist, 1993; Porpino \& Bergqvist, 2002; Porpino et al., 2004, 2014; Zamorano 2012a; Zamorano et al., 2014; Da Costa Pereira et al., 2014).

Castellanos (1942) erigió Panochthus greslebini basándose en fotografías de un tubo caudal procedente del Pleistoceno del Estado de Ceará, noreste de Brasil. Castellanos (1942, p. 583) relata que Mathias G. Oliveira Roxo, de Brasil, le entregó las cuatro fotografías que utilizó para describir su nueva especie, la cual dedicó a su amigo, arqueólogo y arquitecto Héctor Greslebin. En esa misma publicación, Castellanos (1942, p. 570) reconoció P. oliveiraroxoi a base de un tubo caudal proveniente del Estado de Ceará; en la descripción original la nominó como P. oliveira-roxoi (sic), en honor al paleontólogo Mathias G. de Oliveira Roxo, quien también le remitió fotografías y un cuadro de medidas del material mencionado. Posteriormente, Paula Couto (1954) estableció Panochthus rochai a partir de un tubo caudal de similar procedencia geográfica. Tradicionalmente, estas tres formas fueron consideradas como sinónimas (prevaleciendo P. greslebini por prioridad nomenclatural) (Moreira, 1971, p. 551; Porpino \& Bergqvist, 2002, p. 52; ver Zamorano 2012a; Zamorano et al., 2014). Sin embargo, un análisis de Porpino et al. (2014) indica que P. rochai es un sinónimo posterior de $P$. greslebini mientras que $P$. oliveira-roxoi debe ser considerado un nomen dubium. Recientemente, Ferreira et al. (2013) describieron osteodermos atribuidos tentativamente a Panochthus greslebini provenientes, algunos de ellos, del Pleistoceno tardío del Balneario Hermenegildo, Santa Vitoria do Palmars, sur de Brasil (ver Lopes et al., 2010). Fuera del territorio brasileño, el único registro atribuible a P. greslebini corresponde a un tubo caudal fragmentario, procedente del Pleistoceno tardío del Dique "Los Quiroga", Provincia de Santiago del Estero, Argentina (Chimento \& Agnolin, 2011). Sin embargo, la asignación específica de este ejemplar fue recientemente descartada por Porpino et al. (2014).

En esta contribución se da a conocer y se describe detalladamente un tubo caudal completo asignable a Panochthus greslebini, procedente del Lujanense (Pleistoceno tardío) de la margen izquierda del curso inferior del río Quequén Salado, Provincia de Buenos Aires, Argentina (Figura 1). Este constituye el primer hallazgo fehaciente de la especie para la Argentina y a su vez el registro más austral.

\section{CONTEXTO ESTRATIGRÁFICO Y PALEOAMBIENTAL}

Sedimentos correspondientes al Pleistoceno tardío se hallan ampliamente distribuidos a lo largo del curso inferior del río Quequén Salado (Frengüelli, 1928; Alberdi et al., 1989; Farinati \& Zavala, 1995; Pardiñas et al., 1996; Schillizzi et al., 2006; Montalvo et al., 2012; Isla et al., 2014). Estos conforman una potente pila sedimentaria, constituida por niveles conglomerádicos basales, seguidos hacia arriba por una compleja sucesión de limos arcillosos a parcialmente arenosos y areniscas finas, color pardo rojizo a pardo amarillento, con intercalaciones de bancos verde oliva oscuro (ricos en materia orgánica); de estos últimos niveles provienen los restos analizados en la presente contribución (Figura 2).

Estos depósitos, con génesis asociada a un ambiente fluviolacustre, son correlacionables, a base de las características sedimentológicas, antigüedad y fauna de mamíferos presentes, con el Miembro Guerrero de la Formación Luján (Fidalgo et al., 1973) (ver Alberdi et al., 1989) y la Sección media de la Secuencia Agua Blanca (Rabassa, 1989) (ver Farinati \& Zavala, 1995).

Desde una perspectiva bioestratigráfica, los sedimentos portadores son representativos del Piso/Edad Lujanense ( $c a$. 130-8,5 ka AP), habiendo sido reconocidas las biozonas de Equus (Amerhippus) neogeus (ver Cione \& Tonni, 1999, 2001, 2005) у Equus (Amerhippus) neogeus - Macrauchenia patachonica (ver Deschamps, 2005).

Los términos basales de esta sucesión (mayormente representados en la localidad de "Cascada de Paleolama") han sido referidos al interglacial representado por el OIS 5e (130 ka AP) (Transgresión Belgranense) (ver Pardiñas et al., 1996; Cione \& Tonni, 1999, 2001, 2005) mientras que, en proximidades de la desembocadura del río Quequén Salado (Estancia Thomas), sus niveles cuspidales infrayacen, bajo relación de discordancia erosiva, a facies marinas litorales relacionadas con la Transgresión Querandinense (7720 \pm 100 años AP; ver Farinati \& Zavala, 1995).

Se presentan principalmente como rellenos de paleocauces labrados sobre la Serie Neógena [formaciones Irene (Mioceno superior: Piso/Edad Huayqueriense) y La Toma (Plioceno inferior: Piso/Edad Montehermosense) (Oliva et al., 2010; Isla et al., 2014)], siendo a su vez cubiertos por 


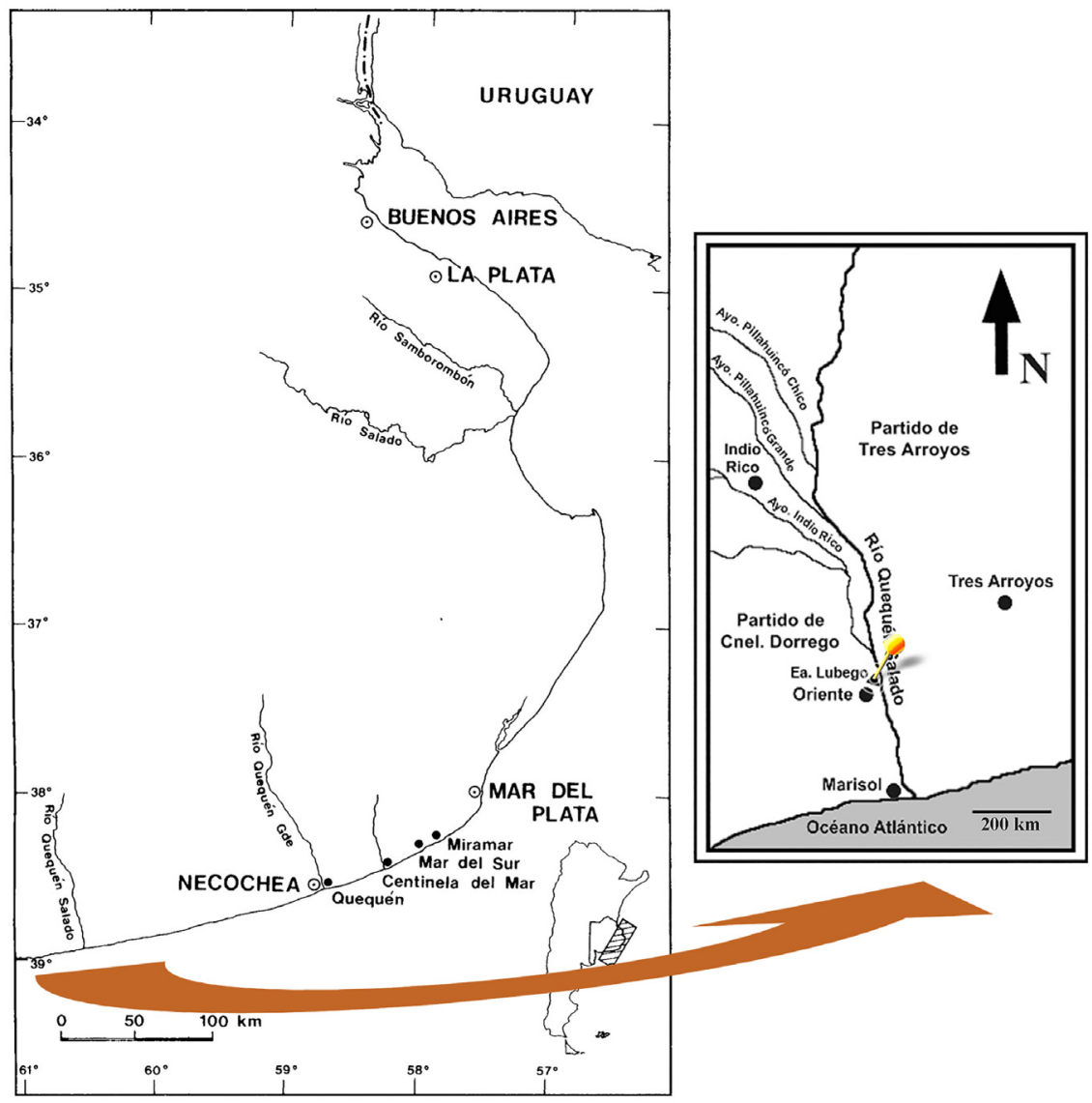

Figura 1. Mapa de ubicación del sitio de hallazgo.

Figure 1. Location map of the outcrop.

niveles arenosos (Frengüelli, 1928), acumulados durante el Holoceno (hasta épocas históricas) en un medio eólico [Miembro Río Salado de la Formación Luján (Fidalgo et al., 1973)], lacustre [Sección superior de la Secuencia Agua Blanca (Rabassa, 1989)] y/o asociados con desbordes [Formación Chacra La Blanqueada (1989)] (ver Frengüelli, 1928; Fidalgo et al., 1973; Farinati \& Zavala, 1995; Isla et al., 2014).

\section{MATERIAL Y MÉTODOS}

Para realizar las descripciones y comparaciones se utilizó la terminología anatómica de Porpino \& Bergqvist (2002) y Zamorano (2012a). Para la sistemática se siguió a Zamorano et al. (2013) (ver Zamorano et al., 2014). Las medidas fueron tomadas con calibre digital y con cinta métrica (ver Figura 3 y Tabla 1).

Abreviaturas institucionales: DGM, Divisão de Geologia e Mineralogia, Rio de Janeiro, Brasil; MACN Pv, Colección Paleovertebrados del Museo Argentino de Ciencias Naturales "Bernardino Rivadavia", Ciudad Autónoma de Buenos Aires, Argentina; MLP, Museo de la Plata, División Paleontología Vertebrados, Facultad de Ciencias Naturales y Museo, Universidad Nacional de La Plata, Buenos Aires, Argentina; MMB, Museo Municipal de
Barreal, departamento de Barreal, San Juan, Argentina; MMH-QUQ, Colección Quequén Salado del Museo Municipal de Ciencias Naturales "Vicente Di Martino", Monte Hermoso, Buenos Aires, Argentina; MNRJ, Museu Nacional, Universidade Federal do Rio de Janeiro, Rio de Janeiro, Brasil; MRFC, Museu Rocha, Fortaleza, Ceará, Brasil; PV-UNS, Paleontología de Vertebrados, Universidad Nacional del Sur, Bahía Blanca, Argentina.

\section{PALEONTOLOGÍA SISTEMÁTICA}

Magnorden XENARTHRA Cope, 1889 Orden CINGULATA Illiger, 1811

Suborden GLYPTODONTIA Ameghino, 1889

Superfamilia GLYPTODONTOIDEA Gray, 1869

Familia GLYPTODONTIDAE Gray, 1869

Tribu HOPLOPHORINI Huxley, 1864

Panochthus Burmeister, 1866

Especie tipo. Glyptodon tuberculatus Owen, 1845.

Panochthus greslebini Castellanos, 1942

1954 Panochthus rochai Paula Couto, p. 195-209, figs. 1-2. 


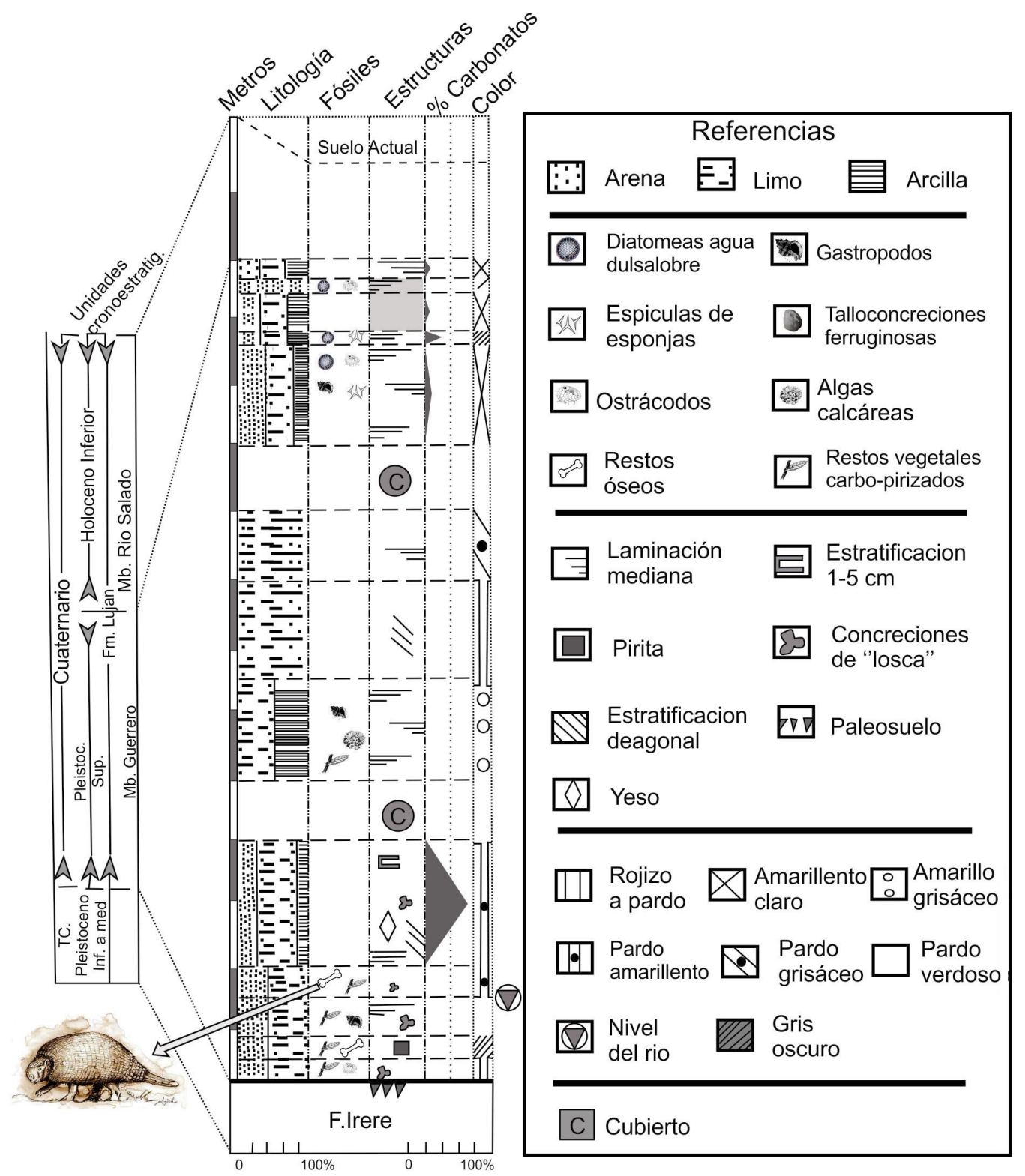

Figura 2. Perfil estratigráfíco de la margen izquierda del río Quequén Salado en la Estancia Lubego (modificado de Schillizzi et al., 2006).

Figure 2. Lithostratigraphical profile from the left side of the Quequé River (modified from Schillizzi et al., 2006).

Holotipo. DGM 1-M [ex SGB1784 (ver Mones, 1986, p. 239)]. Tubo caudal casi completo.

Procedencia estratigráfica y geográfica. "El tubo caudal procede del Estado de Ceará, Brasil, pero se desconoce el horizonte y también los sedimentos que los contenían" (Castellanos, 1942, p. 584). Porpino et al. (2014, p. 1168) precisan que proviene de la ciudad de Jaguaretama, Ceará.

Material referido. El holotipo y MNRJ 2760/1V, tubo caudal; MNRJ 2760/2V, fémur derecho; MNRJ 2760/3V, fémur izquierdo; MNRJ 2760/4V, fragmento de tibia-fíbula derecha; MNRJ 2760/5V, coraza dorsal incompleta; MMH-QUQ 0903-01, tubo caudal completo, en buen estado de preservación (Figura 3). Bergqvist (1989, 1993) (ver Porpino et al., 2014, p. 1169) indica que los restos MNRJ 2760 [divididos por
Bergqvist (1993), /1V, /2V, /3V, /4V y /5V] corresponden a un mismo individuo.

Distribución estratigráfica y geográfica. Pleistoceno del noreste de Brasil, estados de Bahía, Ceará, Paraíba, Pernambuco, Piauí y Río Grande del Norte (ver Da Costa Pereira et al, 2014). Pleistoceno tardío de la Provincia de Buenos Aires, Argentina.

Material aquí presentado. Tubo caudal MMH-QUQ 09-03-01. Procedencia geográfica. Estancia Lubego, margen izquierda del curso inferior del río Quequén Salado, partido de Tres Arroyos, Provincia de Buenos Aires, Argentina (385' S; $60^{\circ} 40^{\prime} \mathrm{O}$ ) (Figura 1).

Procedencia estratigráfica: Unidad Estratigráfica D (Pardiñas et al., 1996) - Unidad 1 (Schillizzi et al., 2006: 


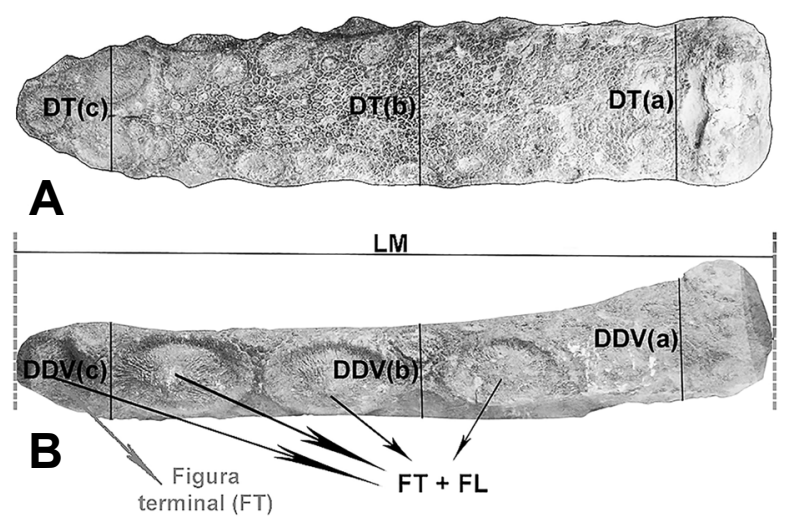

Figura 3. Medidas del tubo caudal. Abreviaturas: DDV, diámetro dorso-ventral del tubo a nivel (a) proximal; (b) medio; (c) distal. DT, diámetro transverso del tubo a nivel (a) proximal; (b) medio; (c) distal. FL, figuras laterales. FT, figura terminal. LM, longitud máxima.

Figure 3. Linear measurements of the caudal tube. Abbreviations: DDV, diameter dorsal-ventral tube (a) proximal; (b) medio; (c) distal. DT, transverse diameter of the tube (a) proximal; (b) middle; (c) distal. FL, lateral figures; FT, terminal figures. LM, maximum size.

Tabla 1. Medidas (mm) de MMH-QUQ 09-03-01, comparadas con DGM 1-M (Panochthus greslebini), MNRJ 2760/IV (P. greslebini), MNRJ 2760/IV (P. greslebini), MACN-Pv 5130 (P. subintermedius), MLP 10-XI-1-1 (P. tuberculatus) y MNRJ 2759/1V (P. jaguaribensis). Simbolos: *, parcialmente tomado de Moreira (1971); ${ }^{\wedge}$, una corresponde al par dorsal-ventral. Abreviaturas: DDV, diámetro dorso-ventral del tubo a nivel (a) proximal; (b) medio; (c) distal. DT, diámetro transverso del tubo a nivel (a) proximal; (b) medio; (c) distal. FL, figuras laterales. FT, figura terminal. LM, longitud máxima.

Table 1. Linear measurements (mm) of MMH-QUQ 09-03-01, compared to DGM 1-M (Panochthus greslebini), MNRJ 2760/IV (P. greslebini), MNRJ 2760/IV (P. greslebini), MACN-Pv 5130 (P. subintermedius), MLP 10-XI-1-1 (P. tuberculatus) and MNRJ 2759/1V (P. jaguaribensis). Symbols: *, partially taken from Moreira (1971); ${ }^{\wedge}$, one corresponding to the dorsal-ventral pair. Abbreviations: DDV, diameter dorsal-ventral tube (a) proximal; (b) medio; (c) distal. DT, transverse diameter of the tube (a) proximal; (b) middle; (c) distal. FL, lateral figures;. FT, terminal figures. LM, maximum size.

\begin{tabular}{lcccccccc}
\hline & LM & DT (a) & DT $(\mathbf{b})$ & DT $(\mathbf{c})$ & DDV $(\mathbf{a})$ & DDV $(\mathbf{b})$ & DDV $(\mathbf{c})$ & FT + FL \\
\hline MMH-QUQ 09-03-01 (P. greslebini) & 810 & 172 & 185 & 173 & $(-)$ & 110 & 98 & 4 \\
DGM 1-M (holotipo de P. greslebini) $\left(^{*}\right)$ & 810 & 175 & 173 & 143 & 141 & 113 & 96 & 4 \\
MNRJ 2760/IV (P. greslebini) & 850 & 179 & 197 & 175 & 160 & 94 & 115 & 4 \\
MACN-Pv 5130 (holotipo de P. subintermedius) & 960 & 198 & 153 & 70 & 201 & 104 & 74 & $\mathrm{I}=4, \mathrm{D}=5$ \\
MLP 10-XI-1-1 (P. tuberculatus) & 780 & 164 & 176 & 106 & 152 & 93 & 82 & 4 \\
MNRJ 2759/1V (P. jaguaribensis) & 985 & 172 & 159 & 96 & 153 & 100 & 80 & $5\left(^{(}\right)$ \\
\hline
\end{tabular}

Perfil D), Lujanense (Pleistoceno tardío-Holoceno temprano) (Figura 2).

Descripción comparada. Morfología general: como es característico de Panochthus greslebini y de las restantes especies de Panochthus conocidas para el lapso BonaerenseLujanense (Pleistoceno medio-Holoceno temprano) $(P$. tuberculatus, $P$. frenzelianus y $P$. jaguaribensis), el tubo caudal presenta un contorno cónico-cilíndrico en su extremo proximal y disminuye su diámetro dorso-ventral en sentido distal; el largo es bastante menor que el de P. subintermedius (Tabla 1). Su diámetro transverso se incrementa ligeramente desde el extremo proximal hasta aproximadamente la mitad de la longitud del tubo, en donde alcanza su máximo desarrollo; desde allí comienza a angostarse ligeramente hasta el margen anterior de las figuras del par dorso-ventral más distal, y disminuye bruscamente desde dicho margen hasta el ápex. Esto lo diferencia de P. subintermedius (Ensenadense, Pleistoceno temprano), en el que el diámetro transverso disminuye paulatinamente desde el extremo proximal hasta el ápex. El tercio proximal tiene forma cónico-cilíndrica, mientras que en los dos tercios restantes es fuertemente deprimido, a semejanza de lo observado en $P$. tuberculatus, P. frenzelianus y P. jaguaribensis. En vista lateral, el tubo presenta una curvatura dorsal a lo largo de toda su extensión, con el extremo distal visiblemente elevado. El ápex de MMH-QUQ 09-03-01 es truncado, similar al del holotipo de $P$. greslebini, aunque sin llegar a experimentar el truncamiento característico de $P$. tuberculatus, $P$. frenzelianus $y$ $P$. jaguaribensis; a su vez, es menos agudo que el del tubo MNRJ 2760/IV. Asimismo, se distingue de P. subintermedius, cuyo ápex es notablemente más agudo. Por último, las dimensiones del material estudiado son similares a aquellas del holotipo de $P$. greslebini (Tabla 1).

Vista dorsal (Figuras 4A,B): el anillo distal (fusionado al tubo) está mal preservado y no es posible apreciar con exactitud su morfología. Por detrás del anillo, aproximadamente a ocho hileras de figuritas periféricas, hay dos hileras transversales de figuras sub-circulares deprimidas, separadas entre sí por 
alrededor de diez hileras de figuritas periféricas. De las dos hileras citadas, las figuras de la hilera proximal son de mayor tamaño y de superficie rugosa; esta hilera se continúa en las caras laterales. La hilera distal está constituida por figuras menores y de superficie más lisa. Al nivel de esta última hilera, comienzan las figuras marginales: estas son depresiones rugosas de contorno sub-circular a elíptico y de diámetro sumamente variable, dispuestas en dos filas sobre los márgenes del tubo, que llegan hasta cerca del borde anterior de las figuras dorsales del par dorso-ventral. También, ubicadas en ambos márgenes, aparecen tres pares de figuras dorso-ventrales (figuras marginales según la nomenclatura de Porpino et al., 2014), de mayor tamaño, más rugosas y excavadas que las figuras marginales; pares de figuras dorsoventrales aumentan de tamaño hacia el extremo distal, el primer par tiene más del doble del tamaño que el tercer par; estas figuras dorso-ventrales están entre las grandes figuras laterales, y se pueden observar también en vista lateral. En la región apical hay dos grandes figuras sub-elípticas, de superficie estriada, situadas simétricamente una al lado de la otra son las figuras dorsales, pertenecientes al par dorsoventral, separadas por un delgado espacio, ocupado por algunas depresiones reducidas y por gran cantidad de figuritas periféricas. Distalmente a las estructuras mencionadas, existe una única figura apical (figura marginal según Porpino et al., 2014), al igual que en $P$. subintermedius y a diferencia de $P$. tuberculatus, $P$. frenzelianus y $P$. jaguaribensis, en las que hay dos de menor diámetro. Es subcircular y de superficie estriada, de tamaño similar a la del holotipo de la especie y mucho mayor (cerca del cuádruple) que la del tubo caudal del holotipo de $P$. oliveiraroxoi (sinónimo de P. greslebini). El resto de la superficie del tubo está cubierto por figuritas periféricas, entre las cuales hay algunas figuras centrales deprimidas, especialmente cerca del ápex.

Vista ventral (Figura 4C): como es característico de todas las especies de Panochthus, se observa un diseño bastante semejante al de la cara dorsal, aunque con las figuras, figuritas, depresiones y rugosidades menos marcadas. Desde esta vista se aprecian las figuras ventrales (pertenecientes al par dorsoventral) y por parte de las figuras laterales y terminales. En el material en estudio no está bien preservada gran parte de la región proximal de la cara ventral (algo menos de 1/3 de la longitud del tubo).

Vista lateral (Figuras 4D,E): ocupando aproximadamente el $75 \%$ de la longitud del tubo hay cuatro grandes figuras subelípticas, deprimidas en la periferia y elevadas en el centro, fuertemente estriadas y delimitadas por rebordes salientes (al igual que en el holotipo de Panochthus greslebini). La más distal de ellas (figura terminal) ocupa toda la superficie lateral y se contacta ventralmente con su correspondiente del otro margen. Las tres figuras restantes (figuras laterales) disminuyen de tamaño proximalmente; la más proximal de estas tiene aproximadamente el $70 \%$ del tamaño de la más distal. También hay cuatro grandes figuras en vista lateral en $P$. tuberculatus y P. frenzelianus, a diferencia de P.jaguaribensis, en el que hay cinco, y de $P$. subintermedius, que presenta cinco $\mathrm{o}$ seis. Anteriormente a las tres figuras laterales (figuras L1-L3 de Porpino \& Bergqvist, 2002 p. 55, fig. 3C), hay una figura sub-circular deprimida y rugosa (Porpino \& Bergqvist, 2002 p. 55, fig. 3C), de tamaño mucho menor. Solo es perfectamente distinguible del lado derecho del tubo, ya que del lado izquierdo la superficie, en esta región, está muy erosionada y no se aprecia bien la ornamentación. Separando estas grandes figuras es posible observar la presencia de numerosas figuritas de morfología similar a la de la coraza dorsal.

Vista posterior (Figura 5): sobre el extremo distal se ubica la figura apexiana, carácter autapomórfico de Panochthus greslebini. Esta figura es también observable en vista dorsal. Es sub-circular, aproximadamente de $14 \mathrm{~mm}$ de diámetro, rugosa y levemente excavada; en cambio, la del holotipo es más circular, de mayor tamaño y más excavada. Está separada de la figura apical de la cara dorsal por una hilera de figuritas.

\section{DISCUSIÓN Y CONCLUSIONES}

Como ya se mencionó, los materiales referidos a Panochthus greslebini proceden del Estado de Ceará y Paraíba. A su vez, la presencia de este taxón ha sido también reportada para los estados de Bahía, Pernambuco, Piauí y Río Grande del Norte (Paula Couto, 1954; Rolim, 1972; Oliveira et al., 1984; Cartelle, 1992; Guérin et al., 1993; Porpino \& Bergqvist, 2002; Da Costa Pereira et al., 2014; Zamorano et al., 2014). Sin embargo, cabe aclarar que los materiales de estos últimos cuatro estados, atribuidos a $P$. greslebini no incluyen el tubo caudal, único material de la especie que exhibe caracteres diagnósticos, por ende esto hace incomprobable dicha asignación específica.

A partir de esta distribución geográfica, Panochthus greslebini fue considerada endémica de esta región (Moreira, 1971; Bergqvist, 1993; Porpino \& Bergqvist, 2002; Porpino et al., 2004; Zamorano 2012a; Da Costa Pereira et al., 2014; Zamorano et al., 2014). No obstante, P. greslebini ha sido citado dos veces fuera de ese territorio. Chimento \& Agnolin (2011, p. 84-88, fig. 3) describen un trozo distal de tubo caudal (MMB-005), procedente del Pleistoceno de Santiago del Estero, Argentina, el cual atribuyen a esta especie. Sin embargo, y en coincidencia con lo sostenido por Porpino et al. (2014), dicho tubo difiere marcadamente del holotipo de P. greslebini por tener la región apical más roma, por carecer de la figura apexiana y por presentar dos figuras subapicales (término empleado por Chimento \& Agnolin, 2011). El ápice agudo, la figura apexiana y la presencia de solamente una figura apical (sin las dos subapicales) son caracteres exclusivos de P. greslebini, presentes tanto en el holotipo como en los materiales atribuidos del NE de Brasil, así como en el tubo caudal que aquí se refiere.

El segundo registro corresponde a Ferreira et al. (2013), quienes describen osteodermos (MNRJ 3537) que atribuyen a Panochthus cf. P. greslebini (sic), provenientes del Estado de Río Grande do Sul, Brasil.

El material que damos a conocer en esta contribución (MMH-QUQ 09-03-01) presenta todos los caracteres propios de Panochthus greslebini: (i) posee una figura apexiana en el extremo distal; (ii) en vista dorsal se encuentra una sola figura 


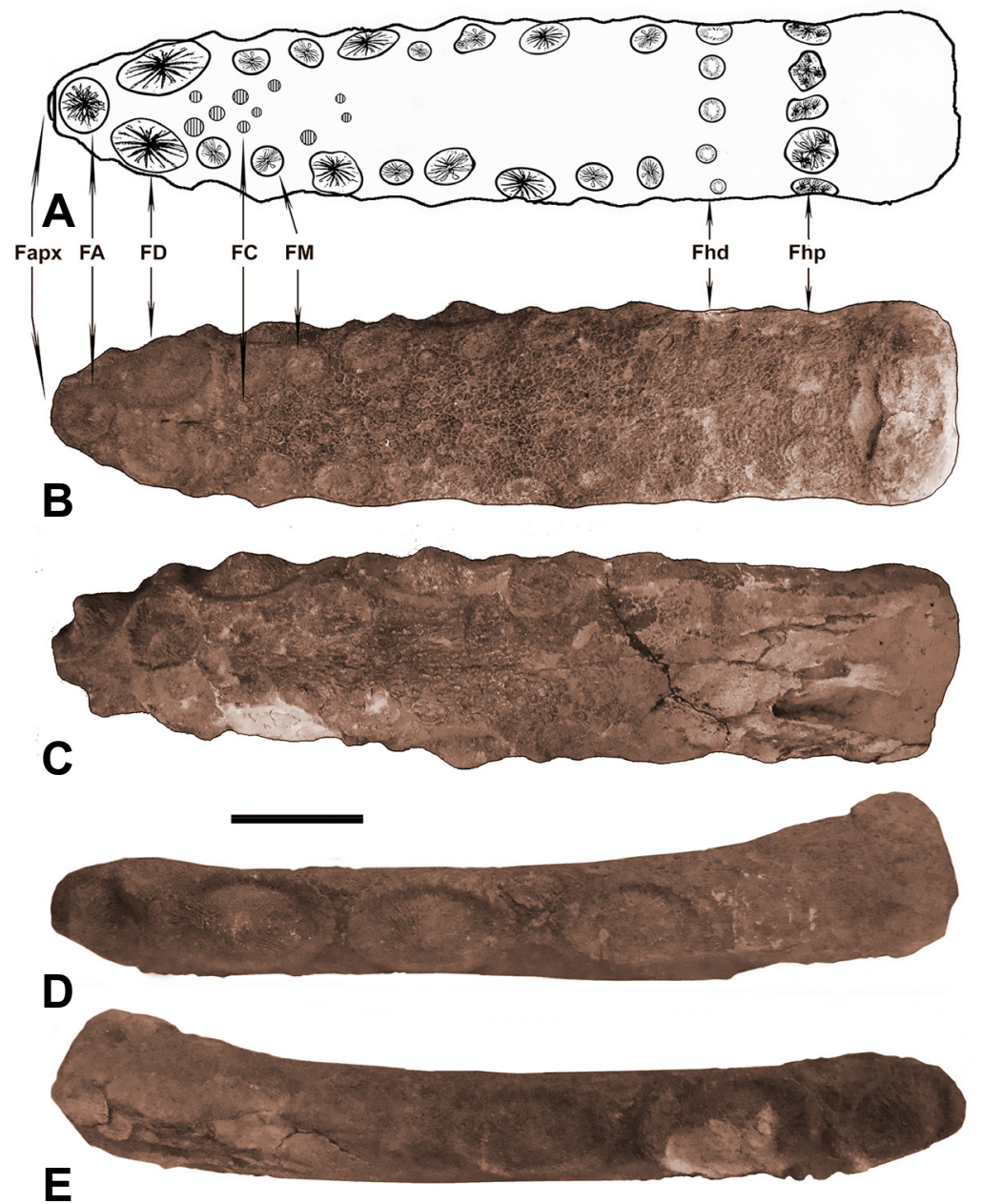

Figura 4. Tubo caudal MMH-QUQ 09-03-01. A, representación esquemática de la vista dorsal; B, vista dorsal; C, vista ventral; D, vista lateral; E, vista lateral izquierda. Abreviaturas: FA, figura apical; Fapx, figura apexiana; FC, figura central; FD, figura dorsal; Fhd, figura hilera distal; Fhp, figura hilera proximal; FM, figura marginal. Escala $=100 \mathrm{~mm}$.

Figure 4. Caudal tube MMH-QUQ 09-03-01. A, schematic representation of the dorsal view; B, dorsal view; C, ventral view; $\mathbf{D}$, right lateral view; E, left lateral view. Abbreviations: FA, apical figure; Fapx, apexian figure; FC, central figure; FD, dorsal figure; Fhd, row distal figure; Fhp, row proximal figure; FM, marginal figure. Scale bar $=100 \mathrm{~mm}$.

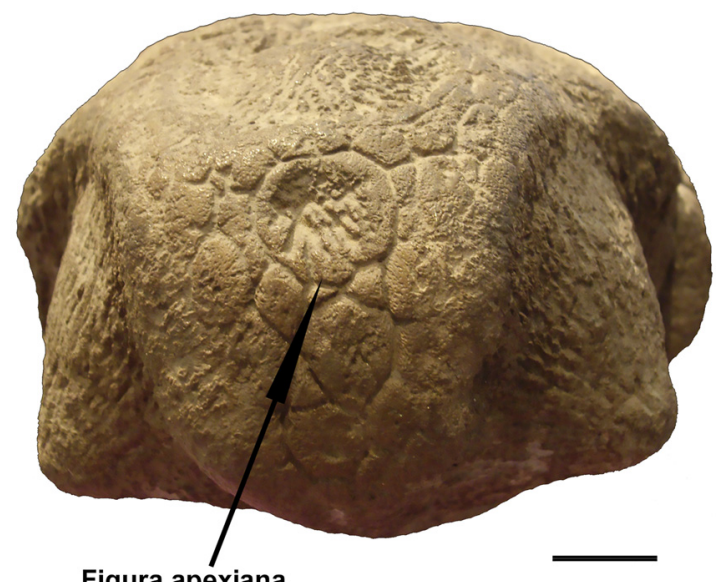

Figura 5. Tubo caudal MMH-QUQ 09-03-01, vista posterior. Escala = $10 \mathrm{~mm}$.

Figure 5. Caudal tube MMH- QUQ 09-03-01, posterior view. Scale bar = $10 \mathrm{~mm}$. 


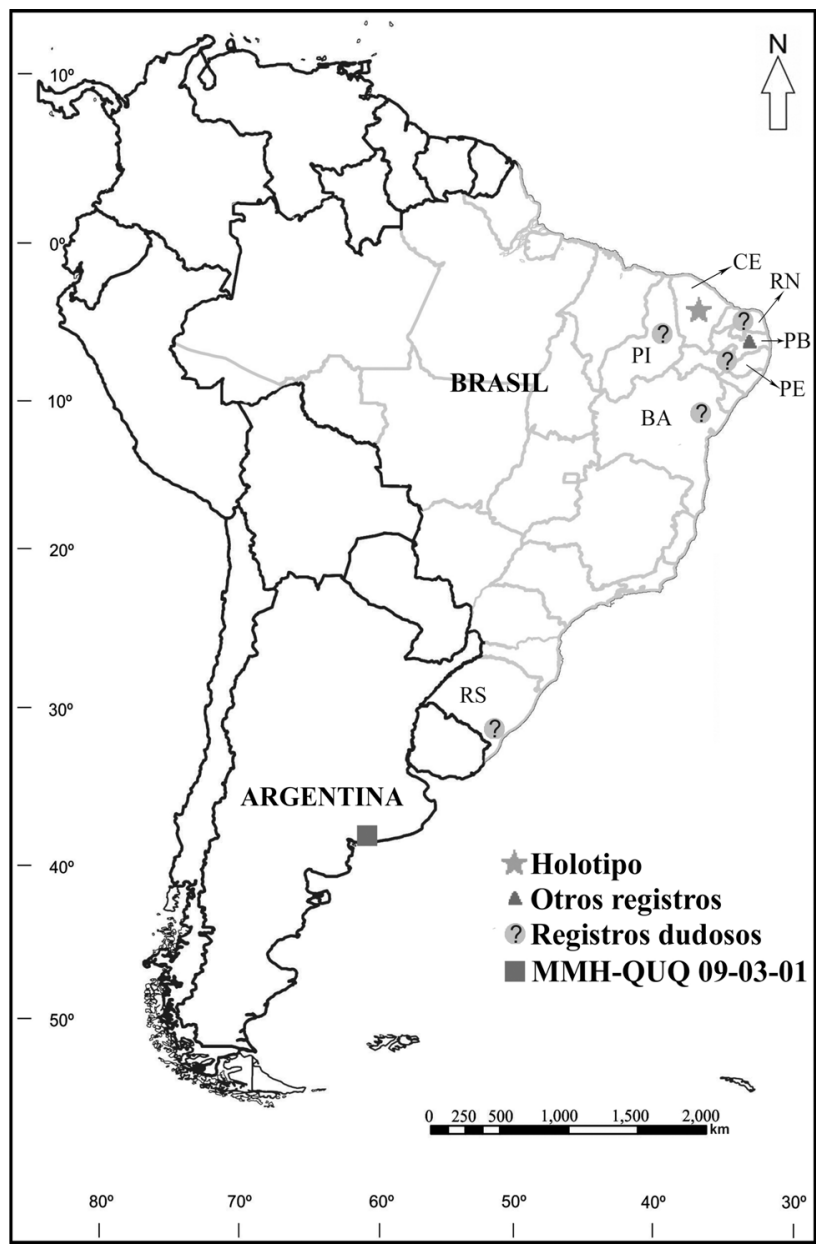

Figura 6. Procedencia geográfica de los materiales atribuidos a Panochthus greslebini. Abreviaturas: CE, Ceará; PB, Paraíba; PE, Pernambuco; RN, Río Grande del Norte; RS, Río Grande del Sur.

Figure 6. Geographic origin of the material assigned to Panochthus greslebini. Abbreviations: CE, Ceará; PB, Paraíba; PE, Pernambuco; RN, Rio Grande do Norte; RS, Rio Grande do Sul.

apical, ubicada distalmente al primer par dorso-ventral, como en $P$. subintermedius, pero en este último el extremo distal es más agudo y el tubo es menos deprimido. Asimismo, se hace notar que este ejemplar, por su morfología general y sus dimensiones, es el más similar al holotipo de todos los tubos hasta ahora atribuidos a la especie.

Desde un punto de vista geográfico, el hallazgo descripto representa el registro más austral de esta especie, ampliando notablemente su distribución latitudinal en más de $3000 \mathrm{~km}$ (Figura 6). De este modo, Panochthus greslebini alcanza la región Pampeana, como ocurre con cuatro de las seis especies válidas de Panochthus, ya que solo P. jaguaribensis no se registra en la región Pampeana (hasta el momento $P$. jaguaribensis es endémica de Brasil). La presencia en la Provincia de Buenos Aires (región Pampeana de Argentina) de $P$. greslebini, taxón hasta ahora exclusivamente brasílico, podría interpretarse en el sentido de que esta especie tuvo un rango amplio de tolerancia ecológica como para extenderse desde Brasil hasta la región Pampeana.

\section{AGRADECIMIENTOS}

A la directora, N. Sánchez, y al personal, M.P. Méder y L. Di Vicente, del Museo Municipal de Ciencias Naturales "Vicente Di Martino", por permitir el acceso al material de estudio y por colaborar en la toma de fotografías y medidas. AN. Chimento y F. Agnolin, por haber facilitado dos fotografías del ejemplar atribuido a Panochthus greslebini, de Santiago del Estero. A los revisores, K.O. Porpino y E.V. Oliveira, y a la editora, Ana María Ribeiro, por enriquecer el trabajo. A J.C. González, por la colaboración en el preparado de las figuras. Este trabajo fue parcialmente financiado por CONICET.

\section{REFERENCIAS}

Alberdi, M.T.; Menegaz, A.N.; Prado, J.L. \& Tonni, E.P. 1989. La Fauna local Quequén Salado - Indio Rico (Pleistoceno tardío) de la provincia de Buenos Aires, Argentina. Aspectos paleo0ambientales y bioestratigráficos. Ameghiniana, 25:225-236

Ameghino, F. 1889. Contribución al conocimiento de los mamíferos fósiles de la República Argentina. Actas Academia Nacional de Ciencias de Córdoba, 6:32-1028.

Bergqvist, L.P. 1989. Mamíferos Pleistocénicos do Estado da Paraíba, Brasil, depositados no Museu Nacional, Rio de Janeiro. Programa de Pós-Graduação em Zoologia, Universidade Federal do Rio de Janeiro, Dissertação de Mestrado, 174 p.

Bergqvist, L.P. 1993. Jazimentos pleistocênicos do Estado da Paraíba e seus fósseis. Revista Nordestina de Biologia, 8:143-158.

Burmeister, G. 1866. Lista de los mamíferos fósiles del terreno diluviano. Anales del Museo Público de Buenos Aires, 1:121-232.

Cartelle, C. 1992. Edentata e megamamiferos herbivoros extintos da Toca dos Ossos (Ourolândia, BA, Brasil) Programa de PósGraduação em Morfologia, Universidade Federal de Minas Gerais, Tese de Doutorado, 516 p.

Castellanos, A. 1937. Los sedimentos del Pampeano inferior y del Araucano en el Valle de Los Reartes (Sierra de Córdoba). Santa Fe, Facultad de Ciencias Matemáticas, Físico-Químicas y Naturales aplicadas a la Industria, 110 p. (Serie TécnicoCientífica 6).

Castellanos, A. 1942. A propósito de los géneros Plohophorus, Nopachthus y Panochthus (3a. parte). Rosario, Universidad Nacional de Rosario, Instituto de Fisiografía y Geología, p. 413-592 (Publicaciones 11).

Chimento, N.R. \& Agnolin, F.L. 2011. Mamíferos del pleistoceno superior de Santiago del Estero (Argentina) y sus afinidades paleobiogeográficas. Papéis Avulsos de Zoologia, 51:83-100. doi:10.1590/S0031-10492011000600001

Cione, A. \& Tonni, E.P. 1999. Biostratigraphy and chronological scale of uppermost Cenozoic in the Pampean area, Argentina. Quaternary of South America and Antartic Peninsula, 12:23-51.

Cione, A. \& Tonni, E.P. 2001. Correlation of Pliocene to Holocene southern South American and European vertebrate-bearing units. Bolletino della Societá Paleontologica Italiana, 40:167-173.

Cione, A. \& Tonni, E.P. 2005. Biostratigrafía basada en mamíferos del Cenozoico superior de la provincia de Buenos Aires, Argentina. In: R. Barrio; M. Etcheverry; M. Capalté \& E. Llambías (eds.) Geología y Recursos Minerales de la Provincia de Buenos Aires, La Plata, p.183-200 (Relatorio 11).

Da Costa Pereira, P.V.L.G.; Victer, G.D.; Porpino, K.O. \& Bergqvist, L.P. 2014. Osteoderm histology of Late Pleistocene cingulates 
from the intertropical region of Brazil. Acta Palaeontologica Polonica, 59:543-552. doi:10.4202/app.2011.0117

Deschamps, C.M. 2005. Late Cenozoic mammal biochronostratigraphy in southwestern Buenos Aires Province, Argentina. Ameghiniana, 42:733-750.

Farinati, E. \& Zavala, C. 1995. Análisis tafonómico de moluscos y análisis de facies en la Serie Holocena del río Quequén Salado, provincia de Buenos Aires, Argentina. In: CONGRESO ARGENTINO DE PALEONTOLOGÍA Y BIOESTRATIGRAFÍA, 6, 1995. Actas, Trelew, APA, p. 117-122.

Fariña, R.A. 2001. Física y Matemáticas para reconstruir la vida en el pasado. Actas de Fisiología, 6:45-70.

Ferreira, J.D.; Zamorano, M. \& Ribeiro, A.M. 2013. Descrição de osteodermos de Panochthus greslebini (Xenarthra, Glyptodontidae) com nova diagnose. In: CONGRESSO BRASILEIRO DE PALEONTOLOGIA, 23, 2013. Resumos, Gramado, SBP, p. 226.

Fidalgo, F.; Colado, U. \& De Francesco, F. 1973. Sobre las ingresiones marinas cuaternarias en los partidos de Castelli, Chascomús y Magodalena (Provincia de Buenos Aires). In: CONGRESO GEOLÓGICO ARGENTINO, 5, 1973. Actas, Neuquén, p. 227-240.

Frengüelli, J. 1928. Observaciones geológicas en la región costanera sur de la Provincia de buenos Aires. Anales de la Universidad Nacional del Litoral, 2:1-145.

Guérin, C.; Curvello, M.A.; Faure, M.; Hugueney, M. \& MourerChauviré, L. 1993. Lá faune pleistocene du Piaui (nordeste du Bresil): implications paleoecologiques et biochronologiques. Quaternaria Nova, 3:303-341.

Hoffstetter, R. 1963. Les glyptodontes du Pléistocène de Tarija (Bolivie). 1: Genres Hoplophorus et Panochthus. Comptes Rendus de la Société Géologique de France, 1963:96.

Hoffstetter, R. 1964. Les glyptodontes du Pléistocène de Tarija (Bolivie). 1: Genres Hoplophorus et Panochthus. Bulletin de la Société Géologique de France, 5:126-133.

Hoffstetter, R. 1978. Une faune de mammifères pléistocènes au Paraguay. Comptes Rendus de la Société Géologique de France, 1978:32-33.

Isla, F.; Dondas, A. \& Oliva, C. 2014. Sedimentología de las Formaciones Irene y La Toma del Neógeno de la Cuenca de Claromecó, Buenos Aires. Geoacta, 39:1-13.

Kerber, L. \& Oliveira, E.V. 2008. Novos Fósseis de Vertebrados para a Sanga da Cruz (Pleistoceno Superior), Alegrete, RS, Brasil. Pesquisas em Geociências, 35:39-45.

Lopes, R.P.; Oliveira, L.K.; Figueiredo, A.M.G.; Kinoshita, A.; Baffa, O. \& Buchmann, F.S.C. 2010. ESR dating of Pleistocene mammal teeth and its implications for the biostratigraphy and geologic evolution of the Rio Grande do Sul coastal plain, southern Brazil. Quaternary International, 212:213-222. doi:10.1016/j.quaint.2009.09.018

Lydekker, R. 1895. Contributions to a knowledge of the fossil vertebrates of Argentina. 2. The extinct Edentates of Argentina. Anales del Museo de La Plata, 3:1-118.

Mones, A. 1986. Paleovertebrata Sudamericana. Catálogo Sistemático de los Vertebrados Fósiles. Parte I (Lista preliminar y bibliografía). Cour. Forsch.-Inst. Senckenbergiana, 82:1-625.

Mones, A. \& Francis, J.C. 1973. Lista de los vertebrados fósiles del Uruguay, 2. Mammalia. Comunicaciones Paleontológicas del Museo de Historia Natural de Montevideo, 1:39-97.

Montalvo, C.; Tomassini, R.; Visconti, G. \& Tiranti, S. 2012. Análisis tafonómico de micromamíferos del Pleistoceno superior del Quequén Salado, provincia de Buenos Aires, Argentina. Revista Brasileira de Paleontologia, 15:3045-358. doi: 104072/ rbp.2012.3.10

Moreira, L.E. 1965. Notas prévias sobre nova espécie de mamífero fóssil do Estado do Caerá, Brasil. Revista de Facultade de Filosofia do Crato, 2:41-49.

Moreira, L.E. 1971. Os gliptodontes do Nordeste do Brasil. Anais da Academia Brasileira de Ciencias, 43:529-552.

Oliva, C.; Di Martino, V.; Dondas, A \& Isla, F. 2010. Paleontología y bioestratigrafía del Neógeno de la cuenca del río Quequén Salado, provincia de Buenos Aires. In: JORNADAS ARGENTINAS DE PALEONTOLOGÍA DE VERTEBRADOS, 25, 2010. Resúmenes, San Luis, p. 25.

Oliveira, L.D.D.; Santos, C.L.A. \& Santos, M.F.C.F. 1984. Nova ocorrência de Panochthus greslebini Castellanos, 1941, no estado do Rio Grande do Norte. In: SIMPÓSIO DE GEOLOGIA DO NORDESTE, 1, 1984. Atas, Natal, UFRN, p. 346-351.

Owen, R. 1845. Descriptive and illustrated catalogue of the fossil organic remains of Mammalia and Aves. London, Museum of the Royal College of Surgeons of London, $391 \mathrm{p}$.

Pardiñas, U.; Gelfo, J.; San Cristobal, J.; Cione, A. \& Tonni, E., 1996. Una asociación de organismos marinos y continentales en el Pleistoceno superior en el sur de la provincia de Buenos Aires, Argentina. In: CONGRESO GEOLÓGICO ARGENTINO, 13, 1993. Actas, Mendoza, p. 95-111.

Paula Couto, C. 1954. Sobre alguns mamíferos fósseis do Ceará. Arquivos do Museu Nacional, 42:195-210.

Porpino, K.O. \& Bergqvist, L.P. 2002. Novos achados de Panochthus (Mammalia, Cingulata, Glyptodontoidea) no Nordeste do Brasil. Revista Brasileira de Paleontologia, 4:51-62.

Porpino, K.O., Fernicola, J.C., Cruz, L.E. \& Bergqvist, L.P. 2014. The intertropical Brazilian species of Panochthus (Xenarthra, Cingulata, Glyptodontoidea): a reappraisal of their taxonomy and phylogenetic affinities, Journal of Vertebrate Paleontology, 34: 1165-1179. doi:dx.doi.org/10.1080/02724634.2014.863203

Porpino, K.O.; Santos, M.C.F.C.; Bergqvist, L.P. 2004. Registros de mamíferos fósseis no Lajedo de Soledade, Apodi, Rio Grande do Norte, Brasil.: Revista Brasileira de Paleontologia, 7:349-358.

Rabassa, J. 1989. Geología de los depósitos del Pleistoceno superior y Holoceno en las cabeceras del río Sauce Grande, provincia de Buenos Aires. In: JORNADAS GEOLÓGICAS BONAERENSES, 1, 1989. Actas, Tandil, p. 765-790.

Rolim, J.L. I972. Paleontologia e estratigrafia do Pleistoceno continental do nordeste brasileiro. "Formação Cacimbas". Programa de Pós-graduação em Geociências, Universidade Federal do Rio Grande do Sul, Dissertação de Mestrado, 119 p.

Schillizzi, R.; Gutierrez Tellez, B. \& Aramayo, S. 2006. Reconstrucción paleoambiental del Cuternario en las barrancas del río Quequén Salado, provincia de Buenos Aires, Argentina. In: CONGRESO ARGENTINO DE CUATERNARIO Y GEOMORFOLOGÍA, 3, 2006. Actas, Córdoba, UNC, p. 649-658.

Soibelzon, L.H.; Zamorano, M.; Scillato-Yané, G.J.; Piazza, D.; Rodríguez, S.; Soibelzon, E.; Tonni, E.P.; San Cristóbal, J. \& Beilinson, E. 2012. Un glyptodontidae de gran tamaño en el Holoceno temprano de la región Pampeana. Revista Brasileira de Paleontologia, 15:113-122. doi:10.4072/rbp.2012.1.09

Tauber, A.A. \& Palacios, M.E. 2007. Nuevos registros de mamíferos cuaternarios de gran porte en la provincia de Santa Cruz, República Argentina. Ameghiniana, 44:41R.

Tonni, E.P.; Soibelzon, E.; Cione, A.L.; Carlini, A.A.; Scillato-Yané, G.J.; Zurita, A.E. \& Paredes Ríos, F. 2009. Preliminar correlation of the Pleistocene sequences of the Tarija valley (Bolivia) with 
the Pampean chronological standard. Quaternary International, 210:57-65. doi:10.1016/j.quaint.2009.06.015

Ubilla, M.; Martinez, S. \& Veroslavsky, G. 2004. El Cenozoico. In: G. Veroslavsky; M. Ubilla \& S. Martínez (eds.) Cuencas sedimentarias de Uruguay: geología, paleontología y recursos naturales, DIRAC-Facultad de Ciencias, p. 11-36.

Zamorano, M. 2012a. Los Panochthini (Xenarthra, Glyptodontidae): Sistemática y evolución. Universidad Nacional de La Plata, Tesis Doctoral, $269 \mathrm{p}$.

Zamorano, M. 2012b. Los Panochthini (Xenarthra, Glyptodontidae): Sistemática y evolución. Mastozoología Neotropical, 19:382-384.

Zamorano, M.; Zurita, A.E. \& Scillato-Yané, G.J. 2013. Contribution to the Systematic of some Glyptodontidae (Xenarthra, Cingulata). In: JORNADAS ARGENTINAS DE PALEONTOLOGÍA DE VERTEBRADOS, 27, 2013. Resúmenes, La Rioja, formato digital.
Zamorano, M.; Scillato-Yané, G.J. \& Zurita, A.E. 2014. Revisión del género Panochthus (Xenarthra, Glyptodontidae). Revista Museo La Plata, 14:1-46.

Zurita, A.E.; Carlini, A.A.; Zamorano, M.; Scillato-Yané, G.J. \& Rivas Duran, B. 2009. Una nueva especie de Panochthus Burmeister (Xenarthra: Glyptodontidae: Panochthini) del Pleistoceno de Bolivia. Ameghiniana, 46:57R.

Zurita, A.E.; Zamorano, M.; Scillato-Yané, G.J.; González-Ruiz, L.R.; Rivas Durán, B. \& Céspedez Paz, R. 2011. An exceptional Pleistocene specimen of Panochthus Burmeister (Xenarthra, Glyptodontoidea) from Bolivia: its contribution to the understanding of the early4-middle Pleistocene Panochthini. Comptes Rendus Palevol, 10:655-664. doi:10.1016/j.crpv.2011.08.002

Received in December, 2014; accepted in July, 2015 